\title{
CD137 Is Required for M Cell Functional Maturation but Not Lineage Commitment
}

\author{
En Hui Hsieh, ${ }^{*}$ Xiomara Fernandez, ${ }^{*}$ Jing Wang, ${ }^{*}$ \\ Mary Hamer, ${ }^{*}$ Stephanie Calvillo, ${ }^{*}$ Michael Croft, ${ }^{\dagger}$ \\ Byoung S. Kwon, ${ }^{\ddagger}$ and David D. Lo*† \\ From the Division of Biomedical Sciences," University of \\ California, Riverside, California; the La Jolla Institute for Allergy \\ and Immunology, ${ }^{\dagger}$ La Jolla, California.; and the Division of Cell \\ and Immunobiology and RED Center for Cancer Therapeutics, ${ }^{\neq}$ \\ National Cancer Center, Ilsan, Goyang-si, Gyeonggi-do, Korea
}

\begin{abstract}
Mucosal immune surveillance depends on $M$ cells that reside in the epithelium overlying Peyer's patch and nasopharyngeal associated lymphoid tissue to transport particles to underlying lymphocytes. $M$ cell development is associated with $B$ lymphocytes in a basolateral pocket, but the interactions between these cells are poorly understood. In a cell culture model of $M$ cell differentiation, we found lymphotoxin/tumor necrosis factor $\alpha$ induction of CD137 (TNFRSF9) protein on intestinal epithelial cell lines, raising the possibility that CD137 on $M$ cells in vivo might interact with CD137L expressed by B cells. Accordingly, while CD137-deficient mice produced UEA-1+ M cell progenitors in nasopharyngeal associated lymphoid tissue and Peyer's patch epithelium, they showed an abnormal morphology, including the absence of basolateral B cell pockets. More important, CD137-deficient nasopharyngeal associated lymphoid tissue $M$ cells were defective in microparticle transcytosis. Bone marrow irradiation chimeras confirmed that while induction of UEA-1+ putative $M$ cell precursors was not CD137-dependent, full $M$ cell transcytosis function required expression of $\mathrm{CD} 137$ by radioresistant stromal cells as well as by bone marrow-derived cells. These results are consistent with a two-step model of $M$ cell differentiation, with initial CD137independent commitment to the $M$ cell lineage followed by a CD137-CD137L interaction of $M$ cells with CD137-activated B lymphocytes or dendritic cells for functional maturation. (Am J Pathol 2010, 177:666-676; DOI: 10.2353/ajpath.2010.090811)
\end{abstract}

The differentiation of lymphoid tissue stromal cells is dependent on complex inducing signals that lead to changes in specific patterns of gene expression among mesenchymal cells, endothelium, and epithelium. One particular apparent paradox in these developmental pathways is the finding that cytokines in the tumor necrosis factor (TNF)/lymphotoxin family are critical to both proinflammatory processes and to differentiation of Iymphoid tissue stroma. Signaling by TNF/lymphotoxin superfamily receptors can activate nuclear factor $\kappa \mathrm{B}(\mathrm{NF}-\kappa \mathrm{B})$ through both the classical (IKK-dependent) and non-classical (relB-dependent) pathways. ${ }^{1}$ Thus, there is no clear distinction between signals that lead to production of inflammatory cytokines versus those that lead to stable development of lymphoid tissue stromal cells such as high endothelial venules or lymphoid mesenchymal cells producing chemokines such as CCL21, 2,3 Chronic production or presentation of TNF/lymphotoxin signals, as in transgenic mice or chronic autoimmune diabetes ${ }^{4}$ may lead to generation of lymphoid structures resembling secondary lymphoid tissue, but it is also possible that controlled combinations of factors may also specify differentiation versus inflammation.

In the case of mucosal lymphoid tissues such as Peyer's patch (PP) and nasopharyngeal associated lymphoid tissue (NALT), in addition to the stromal cells associated with the scaffolding in the lymphoid follicle and high endothelial venule, specific inducing factors are required for the differentiation of the follicle associated epithelium (PPFAE). In the crypts adjacent to the PPAFAE, crypt stem cells are induced by unknown factors to give rise to at least three or more distinct phenotypic subsets: the common follicle associated epithelial cell, occasional goblet cells, and $\mathrm{M}$ cells. ${ }^{5}$ The common follicle associ-

Supported by grants Al63426 (to D.D.L.), Al73689 (to D.D.L.), and Al42944 (to M.C.) from the National Institutes of Health; NCC-0890830-1 and NCCF-0890830-1 from the National Cancer Center, Korea; KRF2005-804-E00001 from the Korean Research Foundation (to B.S.K.), and by a Grand Challenges in Global Health award from the Bill and Melinda Gates Foundation and the Foundation for the National Institutes of Health (to D.D.L).

E.H.H. and X.F. contributed equally to this work.

Accepted for publication April 7, 2010.

Address reprint requests to David D. Lo, M.D., Ph.D., Division of Biomedical Sciences, University of California, Riverside, 900 University Avenue, Riverside, CA 92521. E-mail: david.lo@ucr.edu. 
ated epithelial cell resembles the intestinal enterocyte by morphology (eg, tight junction, brush border microvilli), but recent analysis of gene expression profiling data ${ }^{6-11}$ reveal that these cells show a distinct pattern of gene expression, including expression of unusual extracellular matrix and extracellular matrix-interacting proteins. PPFAE have also been shown to be constitutively positive for the NF- $\kappa B$ gene relB, ${ }^{12}$ which suggests that these cells have persistent activation of NF- $\kappa \mathrm{B}$ signaling, as previously described for dendritic cells which are also relB-positive. ${ }^{13}$ This may be through TNF/lymphotoxin signals provided by follicular lymphocytes; these factors have been implicated in differentiation of secondary and tertiary lymphoid tissue, relying on the alternative NF- $\kappa \mathrm{B}$ pathway. ${ }^{14}$ Moreover, it has been reported that lymphotoxin signaling may be responsible for inducing expression of CCL2O in PPFAE. ${ }^{9,15}$ In this context, Katakai et $\mathrm{al}^{16}$ showed that stromal cell lines would initiate stromal cell like differentiation in the presence of TNF $\alpha$ or $\mathrm{LT} \alpha$, and that even more rapid differentiation would occur in the presence of both TNF $\alpha$ and LT $\beta$ R agonist. Accordingly, we found that treatment of intestinal epithelial cell lines (Caco-2BBe and IEC-6) with LT $\beta R$ agonist and TNF $\alpha$ induced expression of both PPFAE and M cellassociated genes. ${ }^{17}$

Specification of $\mathrm{M}$ cell development is likely to be even more complex than for common PPFAE. It has been suggested that $M$ cells are generated from specialized crypt cell precursors, ${ }^{18}$ but differentiation also requires an association with $B$ lymphocytes that become embedded within a basolateral pocket in the $\mathrm{M}$ cell. ${ }^{19-21}$ So whether or not $\mathrm{M}$ cell precursors are predestined to their phenotype, additional signals from the association with $B$ cells appear to complete the sequence toward final $\mathrm{M}$ cell development. What signals are involved remain unclear; an elegant study by Chervonsky and colleagues ${ }^{22}$ have shown that while $\mathrm{B}$ cells are required for $\mathrm{M}$ cell development, lymphotoxin signals do not have to be directly provided by them, so other, as yet unidentified, B cell factors may be involved. The failure in $\mathrm{M}$ cell development in CCR6-deficient mice ${ }^{23,24}$ also suggest that CCR6-expressing cells, likely recruited by PPFAE expression of CCL20, are important though it remains unclear whether the critical cells are B cells or myeloid cells.

Considering the likely role of lymphotoxin/TNF and $\mathrm{NF}-\kappa \mathrm{B}$ signaling on PPFAE development, we examined the effect of lymphotoxin/TNF treatment on intestinal epithelial cells. The colon carcinoma cell line Caco-2 has been used in co-culture studies as a model for $\mathrm{M}$ cell differentiation, 19,25,26 though in these experiments, the role of lymphotoxin/TNF signaling on gene expression has only recently been studied. ${ }^{9}$ Thus, we studied cytokine effects on Caco-2BBe (a subclone of $\mathrm{Caco}-2^{27}$ ) and the rat IEC-6 ${ }^{28}$ cell lines for the induction of PPFAE and $M$ cell-associated gene expression. ${ }^{17}$ Our data showed up to 60-fold induction of transcription of the TNF receptor superfamily member CD137/4-1BB (TNFRSF9). ${ }^{17}$ This induction by the combination of lymphotoxin and TNF was higher than the induction by either cytokine stimulus alone, suggesting at least an additive effect. We also were able to confirm the report of Rumbo et $\mathrm{al}^{15}{ }^{15}$ that under specific conditions the chemokine CCL20, strongly associated with PPFAE, could be induced by LT $\beta$ R agonist antibody and/or TNF $\alpha$. Most important, the treatment with the combination of TNF $\alpha$ and LT $\beta R$ agonist showed the strongest induction of CD137 transcripts that persisted as long as 72 hours. ${ }^{17}$ This persistent induction of specific genes by combinations of lymphotoxin/TNF ligands may be an important discriminator between their acute proinflammatory effects versus the chronic induction of secondary lymphoid stromal cell development.

Here, we report that the CD137 (4-1BB) protein is also expressed by intestinal epithelial cells in response to TNF/lymphotoxin signaling. In CD137-deficient mice, while the development of mucosal lymphoid tissue appears normal, ${ }^{29,30} \mathrm{M}$ cell functional differentiation is strikingly abnormal, with defects in particle transcytosis by NALT epithelium. Our studies suggest that the development of $\mathrm{M}$ cells in these mice fails at a relatively late stage dependent on the association of immature $M$ cells and basolateral pocket B lymphocytes. This model may provide us with a useful model to study $M$ cell differentiation in which early and late developmental stages may be separated by the expression of and signaling through CD137.

\section{Materials and Methods}

\section{Cell Lines}

Caco-2BBe (ATCC no. CRL-2102) colon carcinoma cells were grown in advanced Dulbecco's modified Eagle's medium (Gibco) with the addition fetal bovine serum to a final concentration of $10 \%$, penicillin-streptomycin-glutamine, and $15 \mathrm{mmol} / \mathrm{L}$ HEPES buffer. T84 (ATCC no. CCL-248) colon carcinoma cell lines were grown in a 1:1 mixture of advanced Dulbecco's modified Eagle's medium and F12 nutrient mixture HAM (Gibco) with fetal bovine serum (5\%), penicillin-streptomycin-glutamine, and $15 \mathrm{mmol} / \mathrm{L}$ HEPES. Cell treatments included addition of goat anti-LT $\beta R$ agonist antibody at $5 \mu \mathrm{g} / \mathrm{ml}$ (R\&D Systems), TNF $\alpha$ at $100 \mathrm{ng} / \mathrm{ml}$ (PeproTech, Inc.) or both. Dose response studies indicated optimal LT $\beta R$ agonist effects (induction of gene expression ${ }^{17}$ ) at doses at or above 2.5 $\mu \mathrm{g} / \mathrm{ml}$, and TNF $\alpha$ responses at or above $50 \mathrm{ng} / \mathrm{ml}$.

\section{Cultures on Transwells}

Cells were cultured on Transwells containing polystyrene supports with a pore size of $0.4 \mu \mathrm{m}$ (Corning) at $10-$ 20,000 cells per well, in a final volume of $600 \mu$ l. Cytokine treatments were done using the concentrations described above. Filters were removed and fixed with PBS/4\% paraformaldehyde for 10 minutes and then rehydrated with PBS for 10 minutes. Casein/PBS (Fisher) with a final concentration of $0.1 \%$ Triton $\mathrm{X}$ was then used for blocking and for dilutions of antibodies. TO-PRO-3 or DAPI was used as a nuclear stain. The slides were post fixed for 5 minutes with $4 \%$ paraformaldehyde, and mounted using ProLong Gold (Molecular Probes). Cells were analyzed using a BD CARV II spinning disk confocal 
microscope, using IPLab software. Image deconvolution and analysis was performed using Volocity software (PerkinElmer).

\section{Animals}

CD137-deficient mice on the BALB/c background were provided by Dr. B.S. Kwon and bred in the UC Riverside vivarium under SPF conditions. In each experiment, wildtype BALB/c age-matched mice were used as controls for CD137-deficient mice. Mice were handled in accordance with institutional IACUC and NIH guidelines.

\section{Bone Marrow Irradiation Chimeric Mice}

Recipient mice were irradiated on day 1 with 1100 rads and were injected with $6-10 \times 10^{6}$ purified bone marrow cells within 20 hours after irradiation. All bone marrow recipient mice received two additional intravenous injections of $40 \times 10^{6}$ purified lymphocytes per injection from PP, LN, and spleen on days 6 and 13 (in proportions of approximately $45 \%$ to $60 \%$ spleen cells, $35 \%$ to $50 \%$ LN, and $1 \%$ to $5 \% \mathrm{PP})$. All bone marrow chimera mice were studied for particle uptake and tissue histology on day 19.

\section{Electron Microscopy}

For Scanning EM, tissues were dissected and fixed in prepared 4\% paraformaldehyde/1\% glutaraldehyde (Electron Microscopy Sciences) and then prepared by critical point drying and gold sputter coating. Samples were examined with a FEI XL30 field emission scanning electron microscopy (SEM) at $5 \mathrm{kV}$. For transmission electron microscopy (TEM), specimens were fixed in $2.5 \%$ glutaraldehyde in $0.1 \mathrm{~mol} / \mathrm{L}$ sodium phosphate, $\mathrm{pH}$ 7.2-7.4, on ice. Tissues were then postfixed with $1 \%$ $\mathrm{OsO}_{4}$ in $0.1 \mathrm{~mol} / \mathrm{L}$ PBS. After gradient ethanol dehydration, samples were infiltrated with Spurr's resin. Ultrathin slices were mounted on Formvar-coated grids and stained with uranyl acetate followed by lead citrate. After air drying, grids were analyzed on an FEI Tecnai-12.

\section{Whole Tissue Confocal Microscopy and Microparticle Uptake Studies}

Dissected NALT or PP tissue was fixed in 1 to $4 \%$ paraformaldehyde/30\% sucrose/PBS, and flash-frozen for cryostat sections, which were then stained according to the protocol described for cell cultures. For whole tissue mounts, dissected tissues were fixed in 1\% paraformaldehyde/30\% sucrose/PBS and then stained in whole tissue fragments. Tissues were mounted in wells built from reinforcement rings on microscope slides for confocal microscopy studies. For microparticle uptake studies, 0.2-micron 505/515 (yellow-green) Neutravidin Fluospheres (Molecular Probes/Invitrogen) were used uncoated (ie, without biotinylated peptide), coated with biotinylated P13 scramble sequence peptide (ie, a Clostridium perfringens enterotoxin (CPE) 30-related se- quence that was scrambled), or coated with biotinylated CPE30 peptide. Under anesthesia, mice were given $6 \times$ $10^{9}$ beads in $40 \mu$ of PBS intranasally (20 $\mu \mathrm{l} /$ nostril), and after 10 minutes, the NALT was dissected for microscopy. For latex bead (Fluoresbrite YG 0.2-micron microspheres; Polysciences) uptake studies in the NALT, mice were given $6 \times 10^{9}$ beads in $40 \mu$ of PBS intranasally (20 microliters/nostril) approximately 1-5 minutes before sacrificing mice for dissection. Tissues were analyzed using a BD CARV II spinning disk confocal microscope, with Volocity software (PerkinElmer) for deconvolution and image analysis. Bead uptake was measured from z-projection images from UEA-1-stained NALT taking $40 \times 40 \mu \mathrm{m}$ areas where UEA-1-positive cells were evident. Each experimental group consisted of at least three individual mice (two NALT per mouse); three non-overlapping confocal z-stacks were taken from each NALT, and three to five non-overlapping $40 \times 40 \mu \mathrm{m}$ image "stamps" were counted from each NALT image. Bead counts were performed using Volocity software. M cell density counts were performed in similar manner. Statistical analysis of bead counts and M cell density were performed by Student's t-test and also by MannWhitney (Prism, GraphPad Software), with similar results.

\section{Results}

\section{TNF/Lymphotoxin Signals and Induction of CD137 in Intestinal Epithelium}

To follow on our observation that CD137 (TNFRSF9/41BB) gene transcripts were induced in Caco-2BBe cells by $\mathrm{LT} \beta \mathrm{R}$ agonist antibody plus $\mathrm{TNF} \alpha,{ }^{17}$ we confirmed that this cytokine treatment also induced expression of CD137 protein as revealed by staining of cytokine treated Caco-2BBe cells both in chamber slides and Transwell cultures (Figure 1). Surprisingly, the CD137 was present in a vesicular distribution within the cytoplasm, and flow

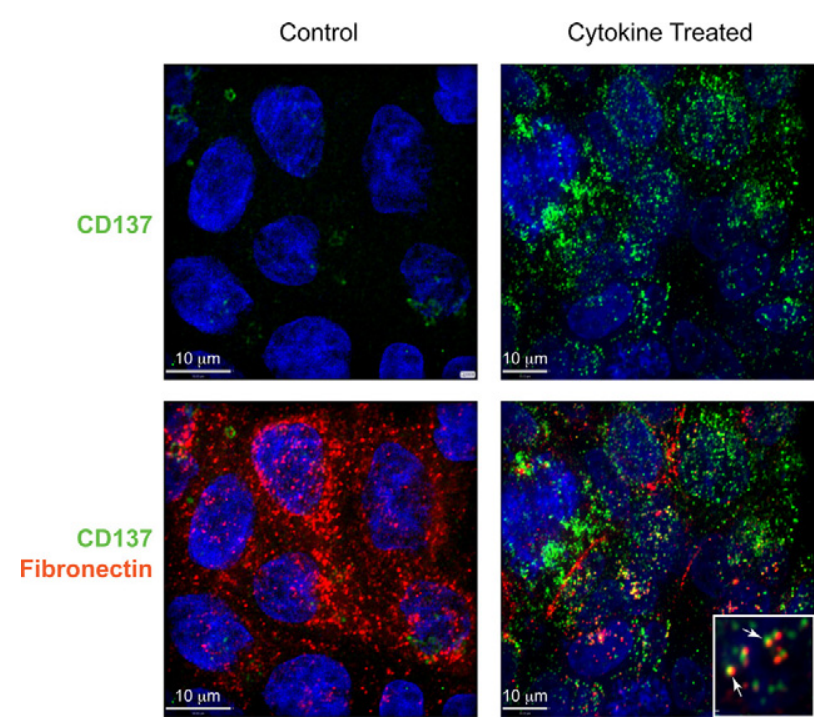

Figure 1. Induction of $\mathrm{CD} 137$ expression in cytokine treated Caco-2BBe cells. Caco-2BBe cells were cultured alone or with a LT $\beta \mathrm{R}$ agonist antibody plus TNF $\alpha$ and stained for CD137 (green) and fibronectin (red). The inset shows co-localization of cytoplasmic CD137 with fibronectin (arrows) 
cytometry analysis could not detect significant levels of cell surface antigen (not shown). The cytoplasmic vesicles with CD137 did not co-localize with the tight junction protein Claudin 4, a protein that we previously reported to be associated with particle endocytosis in cytokinetreated cells. ${ }^{17,31}$ However, we did find that most of the cytoplasmic CD137 co-localized with the extracellular matrix protein fibronectin (Figure 1), though it was not associated with fibronectin at the surface of the cells. The cytoplasmic associations may also account for the apparent decrease in cell-associated fibronectin in the cytokine treated cells; this pattern suggests that fibronectin synthesized by the epithelial cells was endocytosed in association with CD137 either during synthesis or soon after both proteins appeared at the surface of the cells. Interestingly, while CD137 has generally been considered in the context of its expression on lymphocytes and interaction with CD137L expressed on B cells and myeloid cells, ${ }^{29,30}$ two early reports described binding of CD137 to extracellular matrix proteins including laminin, collagen, and fibronectin. ${ }^{32,33}$ It is not known whether CD137 on lymphocytes can bind extracellular matrix and affect lymphocyte migration; however, in the context of intestinal epithelium, it may be more important in the connection of cells to the basement membrane during cytokine-dependent changes, including $\mathrm{M}$ cell differentiation. Whether this interaction also induces signaling though CD137 is not clear.

We attempted histological staining to detect CD137 expression in mouse PPFAE in vivo. While the antibody against human CD137 was able to readily detect the induced protein in the Caco-2BBe cells (Figure 1), with the anti-mouse CD137 antibody we were unable to detect expression in any mouse tissues (not shown). While available antibodies against mouse CD137 could detect the protein on T cells by flow cytometry, they were not sensitive enough to detect CD137 on either T cells or M cells in cryostat sections. Given the much lower level induction in the rat IEC-6 cell line as compared with the human Caco-2BBe line, ${ }^{17}$ it is possible that CD137 expression on mouse mucosal epithelium in vivo is too low to detect or the available reagents are not sensitive enough. Indeed, we have found no published studies showing detection of mouse CD137 by staining mouse tissue sections.

\section{Mucosal Lymphoid Tissues in CD137 Knockout Mice}

\section{Electron Microscopy Studies}

$\mathrm{M}$ cells are most easily found in mucosal lymphoid tissues such as the NALT and PPFAE. Therefore, to assess the importance of CD137 on mucosal M cell development, we examined NALT and PP from mice with a CD137 gene knockout, which had been backcrossed to the BALB/c background. ${ }^{29}$ In previous reports, CD137 knockout mice were not noted to have any unusual developmental defects in any lymphoid tissue, and lymphoid cell populations were noted to be relatively normal. Immune response deficiencies in cytolytic T cells, NK, and NKT cells have been reported, ${ }^{34-36}$ and there have also been reports of alter-
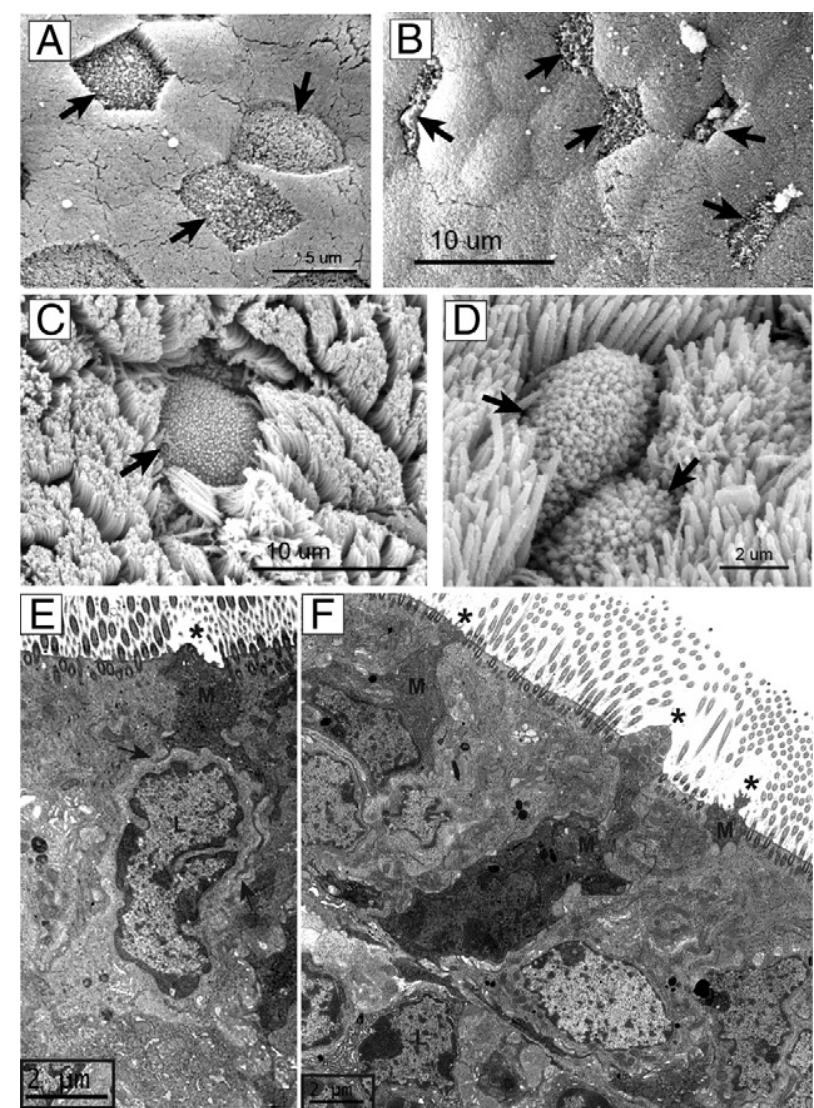

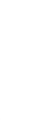

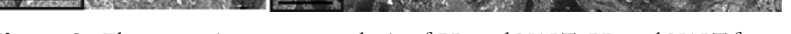

Figure 2. Electron microscopy analysis of PP and NALT. PP and NALT from wild-type $(\mathrm{BALB} / \mathrm{c})$ and CD137-deficient mice were prepared for SEM and TEM to assess the morphology of $M$ cells in mucosal lymphoid tissues. A: SEM of wild-type PP M cells. B: SEM of CD137-deficient PP M cells, showing abnormally narrowed apical surfaces of M cells (arrows). C: SEM of wildtype NALT. D: SEM of CD137-deficient NALT. E: TEM of wild-type NALT, showing $M$ cell (M) lacking apical cilia (asterisk), but with basolateral membranes (arrows) extending around basolateral pocket lymphocyte (L). F: TEM of CD137-deficient NALT, showing narrow cross-section and absence of apical cilia (asterisk) of $\mathrm{M}$ cells (M). Most $\mathrm{M}$ cells lack basolateral lymphocytes; the M cell at upper left may be associated with a lymphocyte, although membrane extensions contacting the lymphocyte are not evident.

ations in T lymphocyte homeostasis and regulation, ${ }^{37}$ including enhanced CD4 T cell responses in vitro, but many aspects of immune responses including antibody production have been reported to be normal, depending on the stimulus. ${ }^{29,38}$ Yet while CD137-deficient mice appear to generate good $\mathrm{T}$ and $\mathrm{B}$ cell responses to systemic or intraperitoneal immunization, specific studies on mucosal immune function have not been reported.

In PPFAE, SEM reveals that most of the epithelial cells have a well defined brush border with tightly packed microvilli uniformly extending less than a micron from the apical surface (Figure 2A). Here, M cells lacking brush border microvilli can be identified across the surface of PP follicle epithelium as gaps in the brush border. The apical surface PP M cells have small projections from the apical surface, while goblet cells have larger projections extending toward the intestinal lumen.

SEM studies of PP revealed a striking phenotype in CD137-deficient mice. Control PP contained clearly identifiable $M$ cells across the entire face of the PPFAE, with M cells showing a characteristic microfold surface clearly 

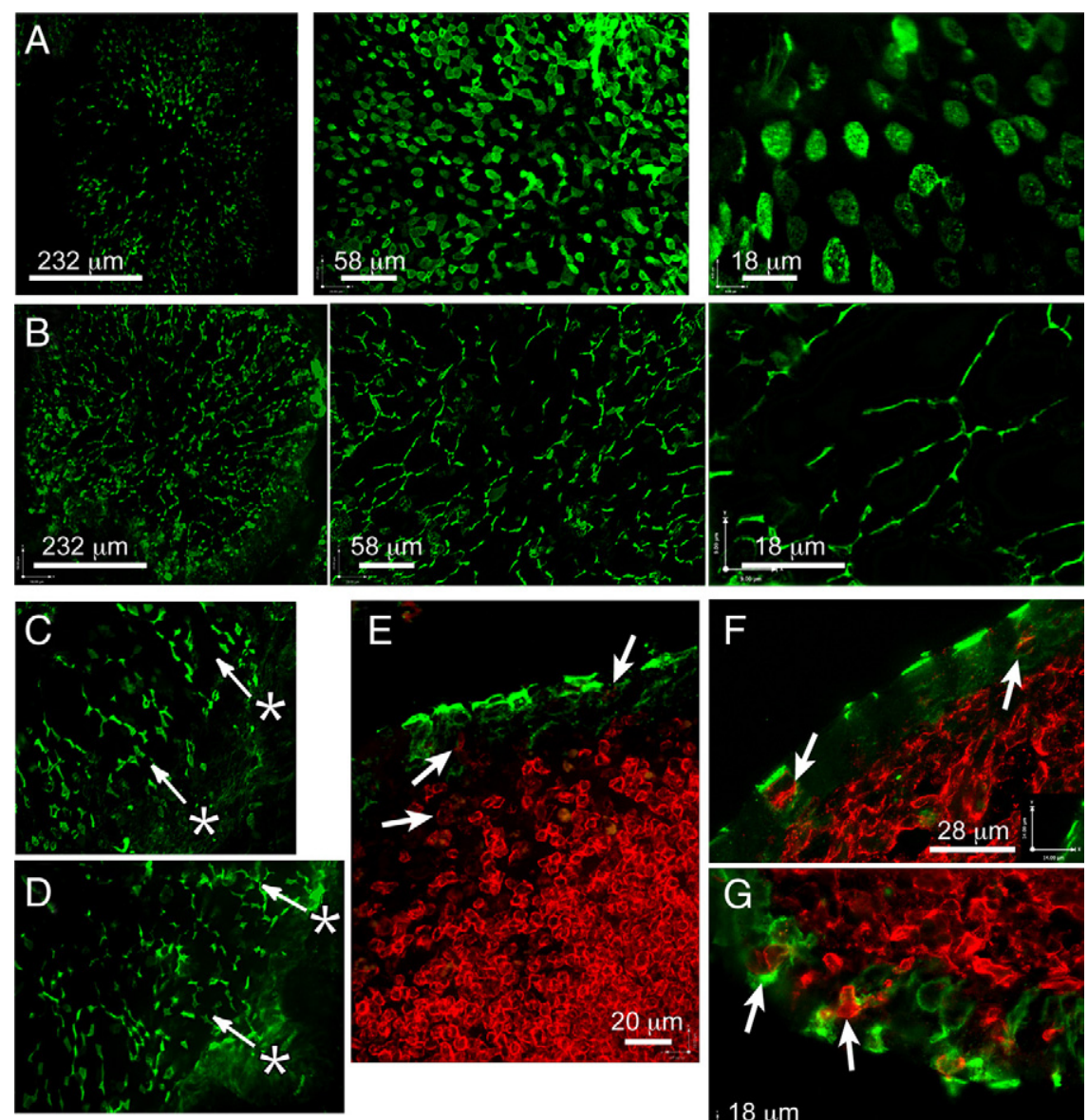
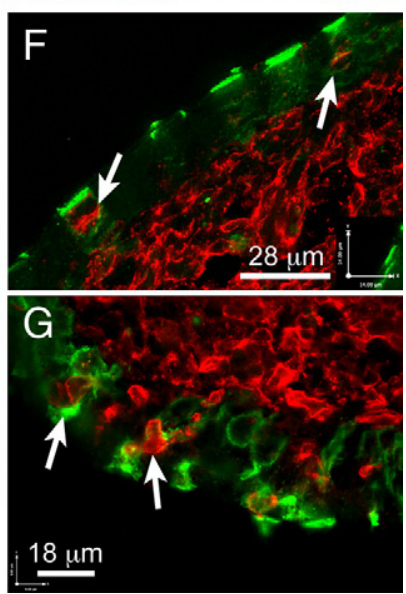

Figure 3. Confocal analysis of Peyer's patch $M$ cells. PP whole mounts were prepared for confocal analysis; serial $z$-sections are projected so that a single follicle could be visualized in a single image at low magnification. A: UEA-1 (green) staining of wild-type PP follicle, with radial pattern of $\mathrm{M}$ cells evident, and at higher magnifications the broad polygonal apical surface is evident. B: UEA-1 staining of CD137deficient PP, showing a similar radial pattern but with a clearly narrowed apical morphology. C: UEA-1 staining of wild-type adjacent crypts, showing pattern consistent with migration of $M$ cells from crypts (asterisk, with arrows indicating apparent course of migration). D: UEA-1 staining of CD137-deficient adjacent crypts (asterisks and arrows as in C). E: B220 (red) versus UEA-1 (green) in wild-type PP, showing weaker expression of B220 by B cells in and near the follicle epithelium (arrows). F: staining MHC class II (red) staining in wild-type PP, showing broad apical surface of UEA-1-positive $\mathrm{M}$ cells (green) and basolateral pocket B lymphocyte (arrows). G: MHC class II staining in CD137-deficient PP, where epithelial MHC II+ cells are evident (red, arrows), but UEA-1-positive $M$ cells (green) are narrower, with less clearly established basolateral pockets. The axis is for scale and orientation. distinguished from the neighboring microvilli of FAE enterocytes (Figure 2A). By contrast, $M$ cells were more difficult to identify in CD137-deficient PP. Presumed M cells (identified provisionally by the lack of brush border microvilli) did not have a clearly flattened polygonal apical surface and instead presented a much smaller apical surface that was also more narrow in shape (Figure 2B).

In the NALT, most of the epithelium displays a dense cover of cilia 2 to $3 \mu \mathrm{m}$ long, so $\mathrm{M}$ cells from wild-type mice were identifiable as cells lacking cilia but having smaller projections from the apical surface (Figure 2C). M cells could be found across NALT from both control and CD137-deficient mice (Figure 2D), but due to the larger number of ciliated cells in the NALT epithelium, differences in the number of distribution of $\mathrm{M}$ cells were not easy to establish. Morphological differences were also difficult to establish, though many of the $M$ cells appeared narrower than in wild-type controls. However, TEM of the NALT from wild-type and control mice showed some differences. Putative $M$ cells in wild-type NALT showed an absence of apical cilia, and in most cases, the basolateral membrane appeared to be intimately associated with an adjacent lymphocyte (Figure 2E, arrows). In NALT from CD137-deficient mice, cells that lacked apical cilia were more difficult to find, with the few cells identified appearing narrow, with most lacking evidence for an associated basolateral lymphocyte (Figure 2F).

\section{Confocal Studies of UEA-1 Lectin Binding}

The distribution of $M$ cells across the face of the PPFAE can also be visualized using whole mount staining of the follicles with the lectin UEA-1. Although goblet cells can also bind UEA-1, they are rare in the PPFAE and NALT epithelium, and they also present a distinctive gobletshaped morphology easily distinguished from conventional $M$ cells. In control PP, UEA-1+ M cells were found in the familiar radial pattern as $\mathrm{M}$ cells are generated from the crypts at the margins of the follicle and migrate to the top of the follicle dome (Figure 3A). As with the SEM images, the apical surface of most wild-type $M$ cells showed a characteristic convex polygonal shape.

Consistent with our findings in the SEM studies, the pattern of UEA-1 staining in CD137-deficient PPFAE was strikingly different from controls. Though UEA-1 cells were evident across the face of the PPFAE, these cells showed a very unusual narrow morphology, consistent with the SEM images in both size and distribution (Figure 3B). The narrow shape was also clearly distinct from goblet cell morphology, so these cells would appear to be in the $\mathrm{M}$ cell lineage, but without full development of the familiar M cell shape. As with control mice, the narrow cells were seen in a radial distribution across the PPFAE, though in some follicles these cells did not get far past the margins of the dome. 
Since $M$ cells in the PPFAE are generated from crypt cell precursors alongside the other PPFAE, we can follow the progression of cells from the crypt of normal and CD137-deficient mice. The pattern of UEA-1-positive cells arrayed in lines extending from the marginal crypt indicates that a crypt cell progenitor has been committed to produce $\mathrm{M}$ cells (Figure $3 \mathrm{C}$ ); the presence of similar radial lines of UEA-1-positive cells in CD137-deficient mice (Figure 3D) suggests that this commitment is normal despite the unusual morphology of cells generated. This phenotype is distinct from the situation in CCR6-deficient mice, in which UEA-1-positive cells were nearly entirely absent; ${ }^{23,24}$ thus, the CD137 knockout appears to affect a later stage in $\mathrm{M}$ cell development than the CCR6 knockout, after commitment of the crypt stem cell to the M cell lineage. Importantly, the absence of CD137 also did not appear to affect their survival in the PPFAE.

\section{Defective Formation of M cell/B Lymphocyte Complexes in CD137-Deficient Mice}

The induction of CD137 on intestinal epithelial cell lines was surprising, since it had previously been described mainly on bone marrow-derived cells such as activated T cells and myeloid cells, ${ }^{39,40}$ though other reports on CD137 expression detected it specifically on vascular endothelial cells in the lung associated with tumors ${ }^{41}$ or associated with atherosclerosis. ${ }^{42}$ The main known ligand for CD137 is the molecule 4-1BBL expressed on B cells and a variety of myeloid cells. Since B cells are known to be important in the induction and maintenance of PPFAE M cells, it was possible that CD137 on developing $\mathrm{M}$ cells is involved in the crosstalk between $\mathrm{M}$ cells and $\mathrm{B}$ cells in the basolateral pocket.

We therefore assessed the morphology of $\mathrm{M}$ cells and associated cells in Peyer's patch follicle epithelium. The narrow morphology of the UEA-1-positive $M$ cells in CD137-deficient mice would appear to be inconsistent with the development of a basolateral pocket with $\mathrm{B}$ lymphocytes that is characteristic of both NALT and PP M cells. Staining for B cell specific markers CD19 and CD45/B220 revealed strong staining on follicular B cells but only very weak staining of the $\mathrm{M}$ cell basolateral pocket cells (Figure 3E, showing B220). By contrast, strong major histocompatibility complex (MHC) class II staining was consistent among $\mathrm{B}$ cells and putative dendritic cells in the subepithelial space. It is possible that the change in B220 and CD19 expression may be a phenotypic change in B cells activated to enter the basolateral pocket. Therefore, in cryostat sections of PP we stained for both UEA-1 to detect M cells and MHC class II antigens (I-A/I-E) to detect putative B cells as well as dendritic cells (Figure 3F). In wild-type BALB/c PP, MHC II-positive cells were found associated with most UEA-1positive $\mathrm{M}$ cells, located in a central-basolateral pocket. The small rounded morphology and fine ring staining for MHC II (arrows) of the basolateral pocket cells was most consistent with their putative identification as B lymphocytes. However, with CD137-deficient mice, while MHC II-positive cells could be found in the follicle epithelium, few if any of these cells were clearly contained within any identifiable M cell basolateral pocket, and their morphology was more irregular (Figure 3G). Thus, it appears that in the absence of CD137, MHC class II-positive cells, whether B cells or dendritic cells, were less efficient in forming stable basolateral pockets or other complexes with UEA-1-positive M cells.

\section{NALTM Cells}

The mouse NALT epithelium is not generated from crypt cell precursors as in the intestine, so there is no radial pattern of UEA-1-positive $M$ cells across the epithelium. Instead, the NALT epithelium of control mice shows a distribution of UEA-1-positive M cells across the NALT. Three-dimensional renderings from confocal z-series showed that these cells were large and more rounded in morphology (Figure 4A). By contrast, the UEA-1-positive $M$ cells in CD137-deficient mice were more irregular in morphology (Figure 4B).

Using confocal z-series of UEA-1 staining cells, we could also construct three-dimensional surface renderings of these cells to assess their morphology. Thus, cells from wild-type NALT showed a roughly cuboid shape, with many displaying a clear basolateral indentation presumably to contain associated lymphocytes (Figure 4C).
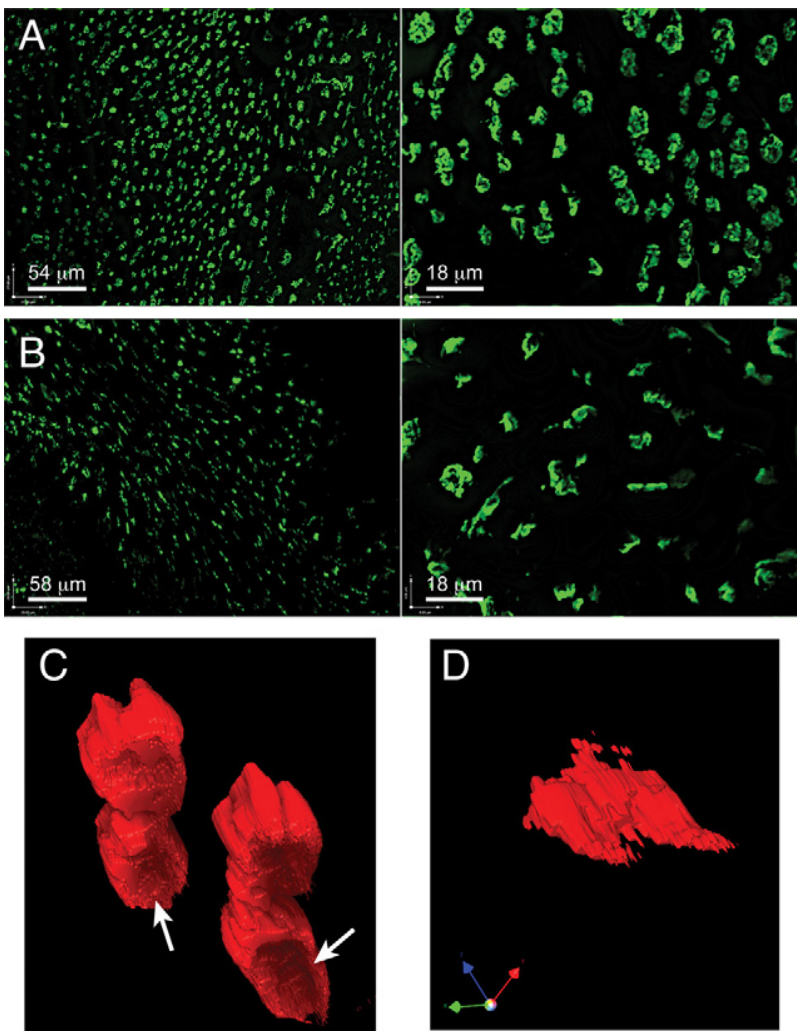

Figure 4. Confocal analysis of NALT M cells. NALT whole mounts were prepared for confocal analysis as described for PP. A: UEA-1 staining of wild-type NALT, showing distribution and broadly rounded apical surface of UEA-1-positive M cells. B: UEA-1 staining of CD137-deficient NALT, showing less uniform morphology of M cells. C: Three-dimensional rendering of wild-type NALT M cell, viewed from below so that the basolateral pocket (arrows) can be seen. D: Three-dimensional rendering of CD137-deficient NALT M cell also viewed from below, showing narrowed morphology. The axis is to show 3-dimensional orientation. 
By contrast, UEA-1-positive cells from the CD137 knockout mice (Figure 4D) showed mainly narrow shaped cells, with no evidence for a basolateral indentation among these cells, consistent with the TEM studies (Figure 2F).

\section{Cell Particle Uptake in NALT and PP}

$\mathrm{M}$ cells, in their role in mucosal immune surveillance, have a specialized capacity for uptake and transcytosis of antigens and microparticles. To test whether the CD137 deficiency had an effect on M cell particle uptake, we relied on the observation that $\mathrm{M}$ cells show regulation and redistribution of the tight junction claudin $4,{ }^{7}$ suggestive of a role for claudin 4 in microparticle uptake. Thus, we took streptavidin-conjugated fluorescent microbeads (0.2 micron diameter) and used them uncoated, or coated them with a control biotinylated peptide, or with a biotinylated 30 amino acid C-terminal peptide from CPE known to bind to an external domain of claudin $4 .{ }^{43,44} \mathrm{We}$ found that NALT M cells were particularly active in microparticle transcytosis; while intestinal PP M cells could be shown to take up particles after hours of lumenal instillation, NALT follicles were found to accumulate microparticles within minutes of administration of a suspension of targeted (CPE peptide-coated) fluorescent styrene microbeads. ${ }^{45}$ This transcytosis appears to be specifically dependent on $\mathrm{M}$ cells; in very brief exposure $(<5$ minutes) to targeted microparticle suspensions, particles found in the wild-type NALT epithelial layer were present within UEA-1+ M cells but many particles were already evident within the lymphoid follicle below. ${ }^{45}$ For more consistent quantitation of particle uptake, bead (both CPE-coated and control peptide-coated) suspensions were instilled into the nasal passages for ten minutes before dissection of the NALT for microscopic analysis of whole mounted tissue (Figure 5, A and B). Control peptide-coated microbeads were found only sparsely taken up in the NALT follicles of wild-type mice. By contrast, large numbers of beads were found taken into the NALT when the beads were coated with the claudin 4-binding peptide (Figure 5C).

In CD137-deficient mice, the uptake of CPE peptidecoated styrene microparticles was far less effective (Figure 5C). Minimal particle uptake was evident using beads coated with control peptide, but more importantly the uptake of beads with claudin 4-binding peptides remained at background levels, and significantly below the uptake by wild-type NALT $(P<0.0001$; Figure 5C). Thus, despite the presence of UEA-1-positive epithelial cells in the CD137-deficient NALT, microparticle uptake was minimal, suggesting that cells committed to $\mathrm{M}$ cell differentiation (evidenced by UEA-1 binding) failed to acquire the capacity to transcytose particles.

Mucosal M cell particle uptake is commonly assessed using latex microbeads; in contrast to protein-coated styrene beads, the apparent low surface charge of these uncoated synthetic particles enables uptake without the need for $\mathrm{M}$ cell targeting ligands (unpublished observations). Thus, we also administered suspensions of 0.2 micron diameter fluorescent latex beads and looked for
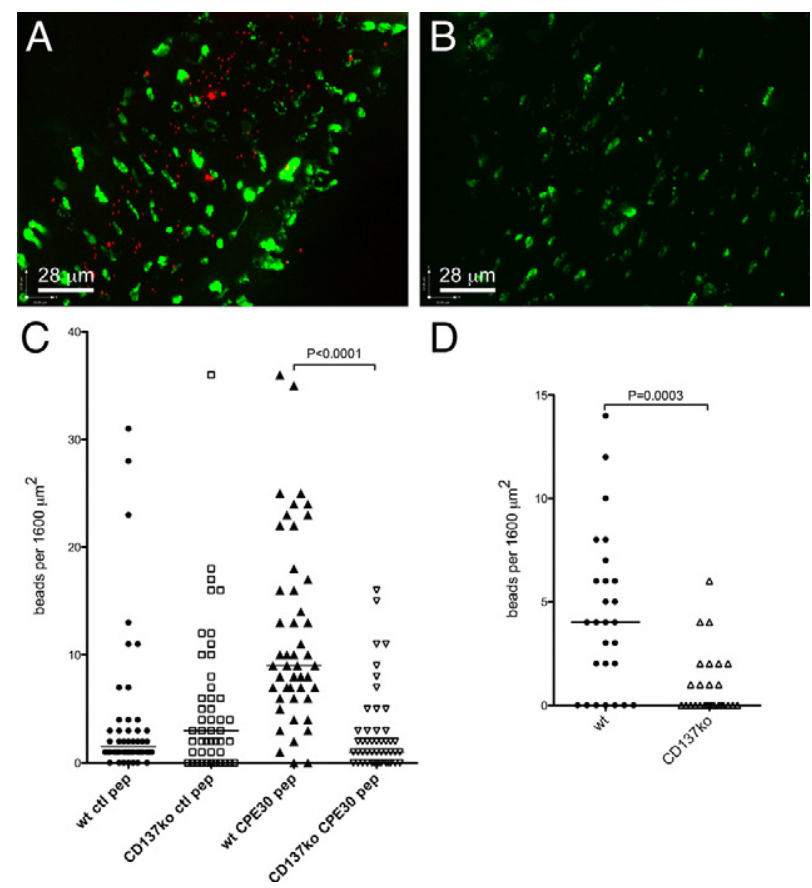

D

Figure 5. Microparticle uptake in NALT. Fluorescent streptavidin-biotinylated peptide-coated styrene (A-C) or uncoated latex (D) microparticles were administered to nasal passages to enable uptake into NALT. A: Uptake of CPE30-coated styrene beads (red) in wild-type NALT, showing that after 10 minutes the beads have already passed the epithelial layer (UEA-1-positive M cells stained green) and entered the follicle. B: Uptake of CPE30-coated beads in CD137-deficient NALT, showing far fewer beads within the follicle. C: Statistical analysis of CPE30-coated bead uptake, showing poor uptake of styrene beads coated with control peptide but significant uptake of CPE30coated styrene beads by wild-type NALT, but little uptake of either type of particle in CD137-deficient NALT. D: Statistical analysis of uncoated latex bead uptake in wild-type and CD137-deficient NALT, showing uptake of beads by wild-type NALT but minimal uptake by CD137-deficient NALT Note that uncoated latex beads are taken up slightly less efficiently than CPE30-coated styrene beads. The axis is for scale and orientation.

particle uptake into the NALT. Here too, we found that uptake of the latex beads in wild-type NALT was significantly greater than uptake by CD137-deficient NALT (Figure 5D).

\section{CD137-Deficient Radiation-Resistant Stroma Supports M Cell Lineage Commitment but Not Function}

CD137 is known to be expressed by bone marrow-derived cells such as T lymphocytes, and our studies have suggested that stromal cells such as mucosal epithelial cells can also be induced to express CD137. Thus, M cell development may require CD137 on bone marrowderived cells, mucosal epithelium, or both. To test this question directly, we made radiation chimeras in which wild-type and CD137-deficient mice were given lethal irradiation, and reconstituted with multiple injections of bone marrow and lymphoid cell populations (spleen, lymph nodes, Peyer's patch) from wild-type or CD137deficient donors ("wild-type $\rightarrow$ wild-type" and "wildtype $\rightarrow$ CD137KO" chimeras, and "CD137KO $\rightarrow$ wildtype" and "CD137KO $\rightarrow$ CD137KO" chimeras). Thus, in these mice the major difference is the CD137 genotype 

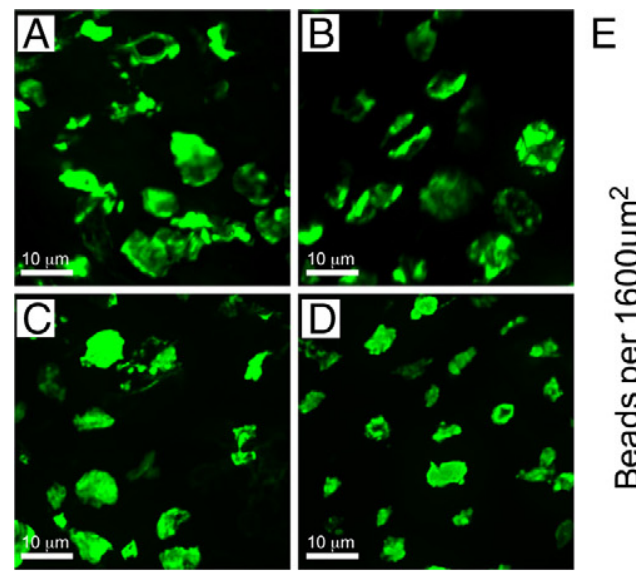

Figure 6. M cell development and function in irradiation chimeras. Irradiation chimeras were analyzed for development of NALT UEA-1+ M cells (A-D), latex particle uptake, and density of M cells (E). Confocal images of representative areas of NALT epithelium showed similar size and distribution of $\mathrm{M}$ cells in all four chimeras: wild-type to wild-type (A); wild-type to CD137KO (B); CD137KO to wild-type (C); CD137KO to CD137KO (D). Among these chimeras, only the wild-type $\rightarrow$ wild-type chimeras showed significant latex particle uptake (E). The density of UEA-1+ M cells across the NALT epithelium was similar for all chimeras $(\mathbf{F})$, suggesting that the defect in uptake was due to functional deficits rather than absence of committed $\mathrm{M}$ cell progenitors.
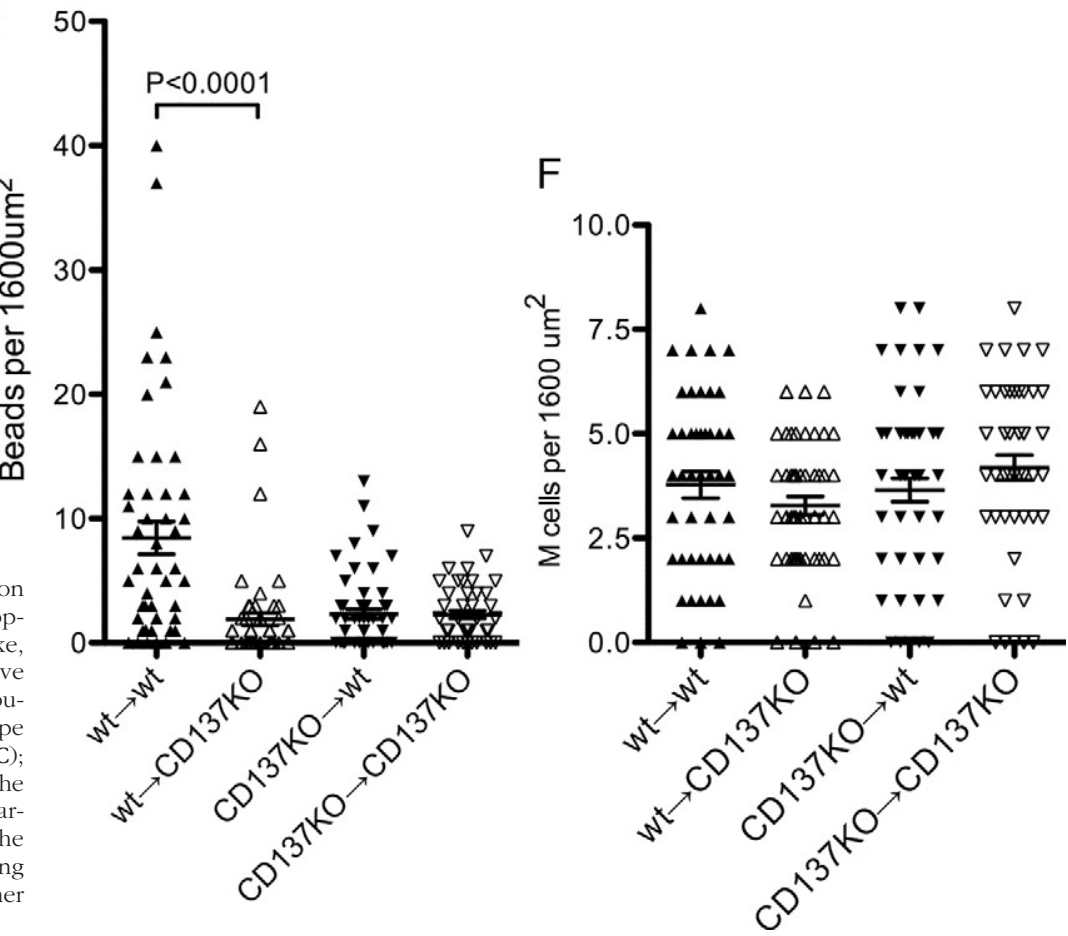

of the bone marrow-derived cells such as lymphocytes and dendritic cells, versus the radioresistant stromal cells, including mucosal epithelium. If CD137 was only required on bone marrow-derived cells (eg, activated $T$ cells and dendritic cells) then only wild-type $\rightarrow$ wildtype and wild-type $\rightarrow$ CD137KO chimeras would be expected to have normal $\mathrm{M}$ cell development and function. If CD137 is only required on stromal cells such as mucosal epithelium, then only wild-type $\rightarrow$ wild-type and $\mathrm{CD} 137 \mathrm{KO} \rightarrow$ wild-type would have normal M cells. A third possibility is that CD137 has two roles, in bone marrow-derived cells and mucosal epithelium; here, only the wild-type $\rightarrow$ wild-type chimeras would have normal $M$ cells.

NALT epithelium in all types of chimeras showed similar development of UEA-1+ M cells (Figure 6, A-D), despite the fact that non-irradiated CD137-deficient NALT showed more irregular M cells (Figure 3); this may be an effect of the irradiation on the epithelium. Fortunately however, when tested for latex microparticle uptake (Figure 6E), the wild-type $\rightarrow$ wild-type chimeras still showed normal particle uptake, indicating that $M$ cell function was present even after irradiation. By contrast, the wild-type $\rightarrow$ CD137KO, CD137KO $\rightarrow$ wild-type, and $\mathrm{CD} 137 \mathrm{KO} \rightarrow \mathrm{CD} 137 \mathrm{KO}$ chimeras all showed very poor to nearly absent microparticle uptake, similar to both unmanipulated CD137-deficient mice and RAG-1-deficient mice (not shown). The difference in the uptake of particle in the NALT of wild-type $\rightarrow$ wild-type versus wild-type $\rightarrow$ CD137KO and other chimeras was highly significant $(P<0.0001)$. Thus, CD137-deficient bone marrow-derived cells failed to support $M$ cell function even in wild-type mucosal epithelium. CD137-deficient mice have been reported to have some $T$ cell functional defects due to a lack of CD137 signaling, ${ }^{29,30}$ so lym- phocyte defects could be responsible in part for the $M$ cell abnormalities. Lymphocyte expression of CD137 is associated with $\mathrm{T}$ cell costimulation, ${ }^{29,34,36}$ so it may be that the connection to M cells is through CD4 T cell interactions with $B$ lymphocytes, which are known to be critical to $\mathrm{M}$ cell development. ${ }^{20,21}$ Interestingly, however, CD137 expression was also required in the radioresistant stromal cells, as wild-type bone marrow failed to induce normal $M$ cell function in the CD137deficient recipients. This would be consistent with a second role for CD137 in the interactions between $B$ cells and $\mathrm{M}$ cells.

As noted in the unmanipulated CD137-deficient mice, both NALT and PP epithelium showed the development of UEA-1+ M cells, although they lacked particle uptake function and had abnormal morphology. In the radiation chimeras, we also saw UEA-1+ presumptive cells in the NALT epithelium. The morphology of the UEA-1+ cells was not strikingly different among the different types of chimeras (Figure 6, A-D); however, the radiation did seem to cause more variable morphology among all of the UEA-1+ cells. Similarly, intestinal Peyer's patches were small and more difficult to examine, likely due to the residual effects of the lethal irradiation. When we counted the distribution of M cells across the NALT epithelium, all chimeras showed a similar density of M cells per surface area of NALT (not significantly different by $t$-test; Figure 6F). Together, these results are consistent with the notion that CD137 is not required for $\mathrm{M}$ cell lineage commitment in mucosal epithelium, but it remains critical for the development of $\mathrm{M}$ cell transcytosis function. 


\section{Discussion}

M cells have been historically difficult to study in detail due to the difficulty in isolating sufficient numbers of cells. Reports on co-cultures of B lymphocytes and Caco-2 cells suggested that this cell culture model could help provide more material for study, but this approach has not been widely used, perhaps due to variations among specific cell line clones available. Moreover, as Kerneis et $\mathrm{al}^{25}$ and Kanaya et $\mathrm{al}^{46}$ have reported, the induction of $\mathrm{M}$ cell-like function in vitro is fairly short-lived (only a few days), and from those studies it is generally not clear what proportion of the cultured cells actually developed $\mathrm{M}$ cell-like function. This perhaps also illustrates the main present limitation in $\mathrm{M}$ cell studies: a clear molecular definition of the $\mathrm{M}$ cell phenotype is only now beginning to be formulated by the collected studies of several groups. Despite these issues, we were able to use the Caco-2BBe and IEC-6 cell lines to assess their response to cytokine induction, and identified genes relating to $\mathrm{M}$ cell development and function. ${ }^{7,17}$ The studies reported here may also provide some additional clues to the stages in $\mathrm{M}$ cell differentiation in mucosal lymphoid tissues. Although lymphotoxin/TNF ligands are known to be important in the development of secondary and tertiary lymphoid tissues, these cytokines are apparently not by themselves sufficient to induce full maturation and function of $\mathrm{M}$ cells in vivo; the physical association with B cells (or subepithelial dendritic cells) may provide additional important signals. ${ }^{20,21}$ Similarly, a recent report suggests that signaling through intestinal epithelium expression of the TNF receptor superfamily member RANK (TNFRSF11a) is also important in M cell differentiation, likely through interaction with the ligand RANKL (TNFSF11) on stromal cells. ${ }^{47}$ Our results suggest that CD137/CD137L signaling between $M$ cell progenitors and basolateral pocket $\mathrm{B}$ cells may provide at least one of these important signals that induce final functional maturation of developing $\mathrm{M}$ cells.

In the radiation chimera studies, results suggest that $\mathrm{M}$ cell function is dependent on CD137 expression by both bone marrow-derived cells and radioresistant stromal cells (presumably epithelial precursors of $\mathrm{M}$ cells). The nature of any lymphocyte-dependent defect (if present) is not yet clear, and lymphoid tissue inducer cells $\left(\mathrm{LTi}^{2}\right)$ are probably normal; the lymphoid tissues of CD137-deficient mice showed overall relatively normal structure and organization, and the development of UEA-1-expressing $M$ cell lineage appeared unimpaired despite their abnormal morphology. The CD137-dependent lymphoid cell contributions to $\mathrm{M}$ cell function may be limited to induction of function after lineage commitment. For example, it may be possible that CD137+ CD4 T cells are involved in inducing B cells to become basolateral pocket lymphocytes in mucosal lymphoid tissues; the basolateral pocket $\mathrm{B}$ cells in turn may be necessary to induce $\mathrm{M}$ cell transcytosis function. In preliminary studies, we found that CD137L triggering increased CCR6 expression in the RajiB cell line (not shown). CCR6 is important in the homing of lymphocytes toward Peyer's patch follicle epithelium expressing the ligand CCL20,9,15,17 and CCR6- deficient mice also have been reported to have $\mathrm{M}$ cell defects, ${ }^{23,24}$ so effects on CD137-induced CCR6 may partly affect $\mathrm{M}$ cell development in the CD137-deficient mice. The requirement for CD137 in radioresistant stroma also suggests a second step in which the recruited CCR6 + CD137L + basolateral pocket B cells interact with CD137 on M cells.

Our histological studies, by both TEM and confocal microscopy, suggested that the CD137-deficient M cells in the NALT and Peyer's patch present a smaller lumenal surface than normal $\mathrm{M}$ cells. The particle uptake defect in these cells could be attributed at least in part to a simple reduction in this apical surface area. However, these morphological differences may be insufficient to explain the functional defect, especially in the radiation chimeras. It may be simpler to suggest that a primary functional defect and failure to form a significant basolateral pocket may also be secondarily associated with the reduced apical surface.

In view of our results, a rough outline of a model might be developed as a working model for M cell differentiation from crypt cell precursors: First, cytokines from lymphoid tissue inducer cells or other follicle associated cells induce nearby crypt cells to convert to $\mathrm{M}$ cell progenitors instead of conventional enterocytes. At this point, the committed progenitors produce the fucose moiety that binds to UEA-1 lectin, and they would also begin to express CD137. Second, a CCR6+ B lymphocyte (perhaps induced by a CD137+ CD4 T cell) migrating to the CCL20+ follicle epithelium encounters the CD137 expressing $M$ cell progenitor, and cross-signaling by CD137/CD137L establishes a bond between the M cell and $B$ cell. Third, the presence of the $B$ cell induces cytoskeletal changes in the $\mathrm{M}$ cell, including formation of the basolateral pocket, and induction of particle transcytosis activity by the $\mathrm{M}$ cell. The B cells in the basolateral pocket might only be for the induction and maintenance of $\mathrm{M}$ cell function, since transcytosed antigens and particles may be more dependent on underlying dendritic cells for presentation to T cells. Thus, basolateral pocket B cells may be a supplementary source of cytokine ligands including lymphotoxin, $\mathrm{TNF} \alpha / \mathrm{LT} \alpha$, and $\mathrm{CD} 137 \mathrm{~L}$, and together these may be responsible for the maintenance of the $\mathrm{M}$ cell functional phenotype. Additionally, the geometry of the basolateral pocket may provide directional clues for transcytosis of particles to the basolateral side of the M cells. CD137 interactions with extracellular matrix proteins may also be important for stabilizing the $\mathrm{M}$ cell contact with the basement membrane, especially since much of the basolateral surface is lost to forming the $\mathrm{B}$ cell pocket. It is interesting to note that the failure of UEA-1+ cells to associate with $B$ cells in CD137-deficient mice does not appear to cause the death of these cells, as they persist across the face of the follicle epithelium dome; thus, B cells do not seem to be needed for any kind of survival signal. The specific effects of CD137-mediated signaling in M cell development is not clear; signaling pathways in CD137+ T cells might be quite different from those mucosal epithelium. However, recent studies have suggested that other non-hemopoietic cells such as vascular endothelium in vivo and 
HUVEC in vitro can be induced to express CD137 by $\mathrm{TNF} \alpha$, and direct triggering of CD137 on HUVEC can induce expression of ICAM- 1 and VCAM- $1 .{ }^{42}$ Similar CD137-induced expression of such adhesion molecules by $M$ cells would help reinforce interactions between $M$ cells and their basolateral pocket $B$ cells expressing receptors such as LFA-1 and VLA-4.

With the specific defect in function by UEA-1-positive cells in CD137-deficient mice, we now have an in vivo system for studying $\mathrm{M}$ cell differentiation in which crypt cells or NALT M cell precursors are able to receive the initial signals that give rise to "immature" M cells (identified by UEA-1 binding), but differentiation fails to proceed past that point. The defect in CD137-deficient M cells also provides us with a tool for dissecting the role of $\mathrm{M}$ cell particle uptake in mucosal immunity.

\section{Acknowledgments}

We thank Lydia Greenlees, Carl Ware, Mick Croft, Paula Norris, Steve Granger, Marian Neutra, Abul Abbas, and Monica Carson for invaluable advice and discussions, and Sharon Santoso, Abby Rynko, and Janelle Crane for technical assistance with these studies. Electron microscopy was performed at the Central Facility for Advanced Microscopy and Microanalysis at UCR.

\section{References}

1. Ware CF: Targeting lymphocyte activation through the lymphotoxin and LIGHT pathways. Immunol Rev 2008, 23:186-201

2. Mebius RE: Organogenesis of lymphoid tissues. Nat Rev Immunol 2003, 3:292-303

3. Cyster JG: Lymphoid organ development and cell migration. Immunol Rev 2003, 195:5-14

4. Kratz A, Campos-Neto A, Hanson MS, Ruddle NH: Chronic inflammation caused by lymphotoxin is lymphoid neogenesis. J Exp Med 1996, 183:1461-1472

5. Neutra MR, Mantis NJ, Kraehenbuhl JP: Collaboration of epithelial cells with organized mucosal lymphoid tissues. Nat Immunol 2001, 2:1004-1009

6. Lo D, Tynan W, Dickerson J, Chang HW, Scharf M, Byrne D, Brayden D, Higgins L, Evans C, O'Mahony DJ: Peptidoglycan recognition protein expression in mouse Peyer's patch follicle associated epithelium suggests functional specialization. Cell Immunol 2003, 224:8-16

7. Lo D, Tynan W, Dickerson J, Scharf M, Cooper J, Byrne D, Brayden D, Higgins L, Evans C, O'Mahony DJ: Cell culture modeling of specialized tissue: identification of genes expressed specifically by follicle-associated epithelium of Peyer's patch by expression profiling of Caco-2/Raji co-cultures. Int Immunol 2004, 16:91-99

8. Hase K, Ihshima S, Kawano K, Hashimoto N, Matsumoto K, Saito H, Ohno $\mathrm{H}$ : Distinct gene expression profiles characterize cellular phenotypes of follicle-associated epithelium and M cells. DNA Res 2005 , 12:127-137

9. Anderle P, Rumbo M, Sierro F, Mansourian R, Michetti P, Roberts M, Kraehenbuhl JP :. Novel markers of the human Follicle-Associated Epithelium identified by genomic profiling and microdissection. Gastroenterol 2005, 129:321-327

10. Verbrugghe P, Waelput W, Dieriks B, Waeytens A, Vandersompele J, Cuvelier CA: Murine M cells express annexin V specifically. J Pathol 2006, 209:240-249

11. Terahara K, Yoshida M, Igarashi O, Nochi T, Soares Pontes G, Hase K, Ohno H, Kurokawa S, Mejima M, Takayama N, Yuki Y, Lowe AW, Kiyono H: Comprehensive gene expression profiling of Peyer's patch $\mathrm{M}$ cells, villous M-like cells, and intestinal epithelial cells. J Immunol 2008, 180:7840-7846
12. Yilmaz ZB, Weih DS, Sivakumar V, Weih F: RelB is required for Peyer's patch development: differential regulation of p52-RelB by lymphotoxin and TNF. EMBO J 2003, 22:121-130

13. Burkly L, Hession C, Ogata L, Reilly C, Marconi LA, Olson D, Tizard $R$, Cate R, Lo D :. Expression of relB is required for the development of thymic medulla and dendritic cells. Nature 1995, 373:531-536

14. Koni PA, Sacca R, Lawton P, Browning JL, Ruddle NH, Flavell RA: Distinct roles in lymphoid organogenesis for lymphotoxins alpha and beta revealed in lymphotoxin beta-deficient mice. Immunity 1997 , 6:491-500

15. Rumbo M, Sierro F, Debard N, Kraehenbuhl JP, Finke D: Lymphotoxin beta receptor signaling induces the chemokine CCL2O in intestinal epithelium. Gastroenterol 2004, 127:213-223

16. Katakai T, Hara T, Sugai M, Gonda H, Shimizu A: Lymph node fibroblastic reticular cells construct the stromal reticulum via contact with lymphocytes. J Exp Med 2004, 200:783-795

17. Wang J, Lopez-Fraga M, Rynko A, Lo DD: TNFR and LTbetaR agonists induce Follicle-Associated Epithelium and $M$ cell specific genes in rat and human intestinal epithelial cells. Cytokine 2009, 47:69-76

18. Gebert A, Posselt W: Glycoconjugate expression defines the origin and differentiation pathway of intestinal M-cells. J Histochem Cytochem 1997, 45:1341-1350

19. Kerneis S, Bogdanova A, Kraehenbuhl JP, Pringault E: Conversion by Peyer's patch lymphocytes of human enterocytes into M cells that transport bacteria. Science 1997, 277:949-952

20. Golovkina TV, Schlomchik M, Hannum L, Chervonsky A:. Organogenic role of B lymphocytes in mucosal immunity Science 1999, 286:1965-1968

21. Mach J, Hsieh T, Hsieh D, Grubbs N, Chervonsky A: Development of intestinal M cells. Immunol Rev 2005, 206:177-189

22. Tumanov AV, Kuprash DV, Mach JA, Nedospasov SA, Chervonsky AV :. Lymphotoxin and TNF produced by B cells are dispensable for maintenance of the Follicle-associated epithelium but are required for development of lymphoid follicles in the Peyer's patches. J Immunol 2004, 173:86-91

23. Lugering A, Floer M, Westphal S, Maaser C, Domschke W, Williams IR, Kucharzik T: Absence of CCR6 inhibits CD4+ regulatory T-cell development and M-cell formation inside Peyer's patches. Am J Pathol 2005, 166:1647-1654

24. Westphal S, Lugering A, von Wedel J, von Eiff C, Maaser C, Spahn T, Heusipp G, Schmidt MA, Herbst H, Williams IR, Domschke W, Kucharzik T: Resistance of chemokine receptor 6-deficient mice to Yersinia enterocolitica infection. Evidence of defective M-cell formation in vivo Am J Pathol 2008, 172:671-680

25. Kerneis S, Caliot E, Stubbe H, Bogdanova A, Kraehenbuhl J, Pringault E: Molecular studies of the intestinal mucosal barrier physiopathology using cocultures of epithelial and immune cells: a technical update. Microbes Infect 2000, 2:1119-1124

26. Gullberg E, Leonard M, Karlsson J, Hopkins AM, Brayden D, Baird AW, Artursson P: Expression of specific markers and particle transport in a new human intestinal M-cell model. Biochem Biophys Res Commun 2000, 279:808-813

27. Peterson MD, Mooseker MS: An in vitro model for the analysis of intestinal brush border assembly. I. Ultrastructural analysis of cell contact-induced brush border assembly in Caco-2BBe cells. J Cell Sci 1993, 105:445-460

28. Quaroni A, Isselbacher KJ, Ruoslahti E: Fibronectin synthesis by epithelial crypt cells of rat small intestine. Proc Natl Acad Sci USA 1978, 75:5548-5552

29. Kwon BS, Hurtado JC, Lee ZH, Kwack KB, Seo SK, Choi BK, Koller $\mathrm{BH}$, Wolisi G, Broxmeyer HE, Vinay DS: Immune responses in 4-1BB (CD137)-deficient mice. J Immunol 2002, 168:5483-5490

30. Moens L, Jeurissen A, Mittler RS, Wuyts G, Michiels G, Boon L, Ceuppens JL, Bossuyt $X$ : Distinct approaches to investigate the importance of the murine 4-1BB-4-1BBL interaction in the antibody response to Streptococcus pneumoniae. J Leukoc Biol 2007, 82:638-644

31. Clark RT, Hope A, Lopez-Fraga M, Schiller N, Lo DD: Bacterial particle endocytosis by epithelial cells is selective and enhanced by tumor necrosis factor-receptor ligands. Clin Vacc Immunol 2009, 16:397-407

32. Chalupny NJ, Peach R, Hollenbaugh D, Ledbetter JA, Farr AG, Aruffo A: T-cell activation molecule 4-1BB binds to extracellular matrix proteins. Proc Natl Acad Sci USA 1992, 89:10360-10364 
33. Loo DT, Chalupny NJ, Bajorath J, Shuford WW, Mittler RS, Aruffo A: Analysis of 4-1BBL and laminin binding to murine 4-1BB, a member of the Tumor Necrosis Receptor Superfamily, and comparison with human 4-1BB. J Biol Chem 1997, 272:6448-6456

34. Lee H-W, Park S-J, Choi BK, Kim HH, Nam K-O, Kwon BS: 4-1BB promotes the survival of $\mathrm{CD} 8+\mathrm{T}$ lymphocytes by increasing expression of Bcl-XL and Bfl-1. J Immunol 2002, 169:4882-4888

35. Vinay DS, Choi BK, Bae JS, Kim WY, Gebhardt BM, Kwon BS: CD137-deficient mice have reduced NK/NKT cell numbers and function, are resistant to lipopolysaccharide-induced shock syndromes, and have lower IL-4 responses. J Immunol 2004, 173:4218-4229

36. Lee S-W, Park Y, Song A, Cheroutre H, Kwon BS, Croft M: Functional dichotomy between OX40 and 4-1BB in modulating effector CD8 T cell responses. J Immunol 2006, 177:4464-4472

37. Lee S-W, Vella AT, Kwon BS, Croft M: Enhanced CD4 T cell responsiveness in the absence of 4-1BB. J Immunol 2005, 174:6803-6808

38. Lee S-C, Ju S-A, Pack H-N, Heo S-K, Suh J-H, Park S-M, Choi B-K, Kwon BS, Kim BS: 4-1BB (CD137) is required for rapid clearance of Listeria monocytogenes infection. Infect Immun 2005, 73:5144-5151

39. Futagawa T, Akiba H, Kodama T, Takeda K, Hosoda Y, Yagita H, Okumura K: Expression and function of 4-1BB and 4-1BB ligand on murine dendritic cells. Intl Immunol 2002, 14:275-296

40. Yang J, Park OJ, Lee YJ, Jung H-M, Woo KM, Choi Y: The 4-1BB ligand and 4-1BB expressed on osteoclast precursors enhance RANKL-induced osteoclastogenesis via bi-directional signaling. Eur J Immunol 2008, 38:1598-1609

41. Zhang G-B, Dong Q-M, Hou J-Q, Ge Y, Ju S-G, Lu B-F, Zhang X-G:.
Characterization and application of three novel monoclonal antibodies against human 4-1BB: distinct epitopes of human 4-1BB on lung tumor cells and immune cells. Tissue Antigens 2007, 70:470-479

42. Drenkard D, Becke FM, Langstein J, Spruss T: Kunz-Schughart LA, Tan TE, Lim YC, Schwarz H: cD137 is expressed on blood vessel walls at sites of inflammation and enhances monocyte migratory activity FASEB J 2007, 21:456-463

43. Takahashi A, Kondoh M, Masuyama A, Fujii M, Mizuguchi H, Horiguchi $\mathrm{Y}$, Watanabe $\mathrm{Y}$ : Role of $\mathrm{C}$-terminal regions of the $\mathrm{C}$-terminal fragment of Clostridium perfringens enterotoxin in its interaction with claudin-4. J Control Release 2005, 108:56-62

44. Ling J, Liao H, Clark R, Wong MSM, Lo DD: Structural constraints for the binding of short peptides to Claudin- 4 revealed by Surface Plasmon Resonance. J Biol Chem 2008, 283:30585-30595

45. Rajapaksa TE, Stover-Hamer M, Fernandez X, Eckelhoefer H, Lo DD: Claudin-4-targeted protein incorporated into PLGA nanoparticles can mediate M cell targeted delivery. J Controlled Release 2010, 142:196-205

46. Kanaya T, Miyazawa K, Takakura I, Itani W, Watanabe K, Ohwada S, Kitazawa H, Rose MT, McConochie HR, Okano H, Yamaguchi T, Aso $\mathrm{H}$ : Differentiation of a murine intestinal epithelial cell line (MIE) towards the $M$ cell lineage. Am J Physiol Gastrointest Liver Physiol 2008, 295:G273-G284

47. Knoop KA, Kumar N, Butler BR, Sakthivel SK, Taylor RT, Nochi T, Akiba $\mathrm{H}$, Yagita $\mathrm{H}$, Kiyono $\mathrm{H}$, Willams IR: RANKL is necessary and sufficient to initiate development of antigen-sampling $\mathrm{M}$ cells in the intestinal epithelium. J Immunol 2009, 183:5738-5747 